\title{
Comprehensive assessment of spotty calcifications on computed tomography angiography: Comparison to plaque characteristics on intravascular ultrasound with radiofrequency backscatter analysis
}

\author{
Joëlla E. van Velzen, $M D,{ }^{a, b}$ Fleur R. de Graaf, $M D,{ }^{a}$ Michiel A. de Graaf, Msc, ${ }^{a}$ \\ Joanne D. Schuijf, PhD, ${ }^{\text {a }}$ Lucia J. Kroft, $\mathrm{MD}, \mathrm{PhD},{ }^{\mathrm{c}}$ Albert de Roos, $\mathrm{MD}, \mathrm{PhD},{ }^{\mathrm{c}}$ \\ Johan H. C. Reiber, PhD, ${ }^{\mathrm{c}}$ Jeroen J. Bax, MD, PhD, ${ }^{\mathrm{a}}$ J. Wouter Jukema, MD, PhD, ${ }^{\mathrm{a}, \mathrm{b}}$ \\ Eric Boersma, PhD, ${ }^{d}$ Martin J. Schalij, MD, PhD, ${ }^{a}$ \\ and Ernst E. van der Wall, MD, PhD ${ }^{\mathrm{a}, \mathrm{b}}$
}

Background. The purpose of the study was to systematically compare calcification patterns in plaques on computed tomography angiography (CTA) with plaque characteristics on intravascular ultrasound with radiofrequency backscatter analysis (IVUS-VH).

Methods and Results. In total, 108 patients underwent CTA and IVUS-VH. On CTA, calcification patterns in plaques were classified as non-calcified, spotty or dense calcifications. Plaques with spotty calcifications were differentiated into small spotty $(<1 \mathrm{~mm})$, intermediate spotty $(1-3 \mathrm{~mm})$ and large spotty calcifications $(\geq 3 \mathrm{~mm})$. Plaque characteristics deemed more high-risk on IVUS-VH were defined by \% necrotic core (NC) and presence of thin cap fibroatheroma (TCFA). Overall, 300 plaques were identified both on CTA and IVUS-VH. \% NC core was significantly higher in plaques with small spotty calcifications as compared to noncalcified plaques $(20 \%$ vs $13 \%, P=.006)$. In addition, there was a trend for a higher $\% \mathrm{NC}$ in plaques with small spotty calcifications than in plaques with intermediate spotty calcifications $(20 \%$ vs $14 \%, P=.053)$. Plaques with small spotty calcifications had the highest \% TCFA as compared to large spotty and dense calcifications $(31 \%$ vs $9 \%$ and $31 \%$ vs $6 \%, P<.05)$.

Conclusion. Plaques with small spotty calcifications on CTA were related to plaque characteristics deemed more high-risk on IVUS-VH. Therefore, CTA may be valuable in the assessment of the vulnerable plaque. (J Nucl Cardiol 2011;18:893-903.) plaque

Key Words: Atherosclerosis • computed tomography $(\mathrm{CT}) \cdot$ vulnerable atherosclerotic

\section{INTRODUCTION}

Computed tomography angiography (CTA) is a rapidly evolving technique that has the ability to noninvasively and accurately detect significant coronary artery stenosis and coronary atherosclerotic plaque. ${ }^{1-5} \mathrm{~A}$ potentially interesting application of CTA would be the

From the Department of Cardiology, ${ }^{\mathrm{a}}$ and Department of Radiology, ${ }^{\mathrm{c}}$ Leiden University Medical Center, Leiden, The Netherlands; The Interuniversity Cardiology Institute of the Netherlands, ${ }^{\text {b }}$ Utrecht, The Netherlands; and Department of Cardiology, ${ }^{\mathrm{d}}$ Erasmus Medical Center, Rotterdam, The Netherlands.

Funding: Dr van Velzen is supported by the Dutch Heart Foundation (The Hague, The Netherlands), Grant nr. 2007B223. Dr de Graaf is co-supported by the Dutch Technology Foundation STW (Utrecht, The Netherlands), applied science division of I and the Technology Program of the Ministry of Economic Affairs, Grant nr. 10084. identification of patients or lesions that have an increased likelihood of plaque rupture leading to acute coronary events. Several previous studies have identified specific plaque characteristics which are frequently observed with CTA in patients presenting with acute coronary syndrome (ACS). ${ }^{6-8}$ Among these characteristics, a

Received for publication Mar 18, 2011; final revision accepted Jun 28, 2011.

Reprint requests: Ernst E. van der Wall, MD, PhD, Department of Cardiology, Leiden University Medical Center, Postal Zone: C5-P, P.O. Box 9600, 2333 ZA Leiden, The Netherlands; e.e.van_der_ wall@lumc.nl.

$1071-3581 / \$ 34.00$

Copyright (C) 2011 The Author(s). This article is published with open access at Springerlink.com

doi:10.1007/s12350-011-9428-2 
spotty pattern of calcifications has been related to the presence of ACS. ${ }^{6-8}$ Indeed, preliminary data with CTA demonstrated that culprit lesions in patients with ACS had a higher prevalence of smaller spotty calcifications than target lesions in patient with stable complaints. ${ }^{9}$ Moreover, the presence of spotty calcifications on CTA were associated with a higher likelihood of developing ACS. ${ }^{8}$

More detailed information on plaque characteristics can be obtained by intravascular ultrasound with radiofrequency backscatter analysis (IVUS-VH). ${ }^{10,11}$ This technique was developed to improve grayscale intravascular ultrasound tissue characterization and provide detailed quantitative information on plaque composition in vivo. ${ }^{12,13}$ Additionally, the presence of thin cap fibroatheroma (TCFA) on IVUS-VH was demonstrated to be independently predictive of major cardiovascular events during follow-up. ${ }^{14}$

However, the relation between calcification patterns on CTA and plaque characteristics on IVUS-VH has not been previously reported. Furthermore, in previous studies, the definition of spotty calcification on CTA has been variable. Indeed, definitions for spotty calcification on CTA have ranged from presence of any calcified material embedded in non-calcified plaque to a specific threshold regarding the size of calcification. Subsequently, due to the inconsistency of definitions, comparability of spotty calcifications on CTA between investigations is hampered. Therefore, the purpose of the study was to perform a systematic evaluation of plaques with different calcification patterns on CTA and relate this to plaque characteristics on IVUS-VH.

\section{METHODS}

\section{Patient Population and Study Protocol}

A total of 108 patients were included in this evaluation who underwent CTA prior to invasive coronary angiography with IVUS-VH of 1 to 3 vessels, as part of clinical protocol. For this retrospective evaluation, consecutive patients were selected as part of an ongoing registry addressing the relative merits of CTA in relation to other imaging techniques. ${ }^{15}$ Patient with chest pain were referred for imaging for the noninvasive evaluation of coronary artery disease (CAD), according to clinical protocol. Thereafter, patients were referred for invasive coronary angiography in combination with IVUS-VH based on the patient's clinical presentation and/ or imaging results to further evaluate the extent and severity of CAD. During invasive coronary angiography, an experienced interventional cardiologist decided whether IVUS studies should be performed. Clinical history was evaluated between the CTA examination and invasive coronary angiography to ensure that neither acute coronary syndromes nor worsening of angina occurred between examinations. Exclusion criteria for CTA examination were: (i) (supra) ventricular arrhythmias, (ii) renal failure (glomerular filtration rate $<30 \mathrm{~mL} /$ minute), (iii) known allergy to iodine contrast material, (iv) severe claustrophobia, (v) pregnancy. Patient data were prospectively collected in the departmental Cardiology Information System (EPD-Vision ${ }^{\circledR}$, Leiden University Medical Center, Leiden, the Netherlands) and retrospectively analyzed. Our institutional review board does not require its approval and written informed consent for retrospective technical analysis of data, as was the case for this study. In each patient, the presence of risk factors for $\mathrm{CAD}$ and medication (use of aspirin, statins, beta-blocking medication and angiotensin-converting enzyme (ACE) inhibitors) were recorded.

\section{CTA}

Image acquisition. CTA examination was performed using either a 64-row helical scanner (Aquilion 64, Toshiba Medical Systems, Toshiba Medical Systems, Otawara, Japan) or a 320-row volumetric scanner (Aquilion ONE, Toshiba Medical Systems, Otawara, Japan). If the patient's heart rate was $\geq 65$ beats/minute and no contra-indications existed, beta-blocking medication (metoprolol 50 or $100 \mathrm{mg}$, single oral dose, 1 hour prior to examination) was administered. Scan parameters for the 64-row contrast enhanced scan were: $400 \mathrm{~ms}$ gantry rotation time, collimation of $64 \times 0.5 \mathrm{~mm}$, tube voltage of $100-135 \mathrm{kV}$ and tube current of 250-350 mA, depending on body posture. Non-ionic contrast material (Iomeron 400, Bracco, Milan, Italy or Ultravist 370, Bayer Schering Pharma AG Berlin, Germany) was administered with an amount of $80-110 \mathrm{~mL}$ followed by a saline flush with a flow rate of $5 \mathrm{~mL} / \mathrm{second}$. Datasets were reconstructed from the retrospectively gated raw data, the best phase was reconstructed with an interval of $0.3 \mathrm{~mm}$. Using a single test slice reconstructed throughout the various phases of the heart cycle, other suitable R-R intervals were examined for additional reconstructions. Concerning the 320-row contrast enhanced scan; imaging was performed in a single volume using prospective triggering with exposure interval depending on the heart rate. If the heart rate was $\geq 65$ beats/minute, the phase window was set $65 \%-85 \%$ of R-R interval, if the heart rate was stable and $<60$ beats/minute the phase window was set at $70 \%-80 \%$ of R-R interval. Scan parameters were: $350 \mathrm{~ms}$ gantry rotation time, $320 \times 0.5 \mathrm{~mm}$ collimation, 100 $135 \mathrm{kV}$ tube voltage and a tube current of $400-580 \mathrm{~mA}$, depending on body mass index (BMI). Overall, 60-90 mL contrast material (Iomeron 400, Bracco, Milan, Italy) was administered with a rate of 5-6 mL/second followed by a saline flush. Data acquisition was performed during an inspiratory breath hold of approximately 4-10 seconds. Subsequently, images were reconstructed in the best phase of the $\mathrm{R}-\mathrm{R}$ interval and transferred to a remote workstation for postprocessing.

Image analysis. CTA datasets were evaluated using dedicated software (Vitrea 2.0 or Vitrea FX 1.1 Vital images, Minnetonka, MN, USA). Analysis was performed with the use 
of available post-processing tools such as cross-sectional axial slices and multiplanar reconstructions. One experienced reader, blinded to the IVUS-VH results, evaluated the CTA datasets. The coronary arteries were divided into 17 segments according to the modified American Heart Association classification. ${ }^{16}$ Each segment was evaluated for the presence of any atherosclerotic plaque. Structures $>1 \mathrm{~mm}^{2}$ within and/or adjacent to the coronary artery lumen, which could be clearly distinguished from the vessel lumen, were defined as atherosclerotic plaque. ${ }^{3}$

Calcification patterns in plaques on CTA were classified morphologically as non-calcified, spotty calcifications or dense calcifications. Non-calcified plaques were defined as a plaque with low CT attenuation located in the vessel wall in at least two independent image planes and clearly distinguishable from the contrast-enhanced lumen and pericardial tissue without any calcification. ${ }^{17}$ Plaques with spotty calcifications were defined as follows: length (extent in the longitudinal direction of the vessel) of the calcification $<3 / 2$ of vessel diameter and width (extent of the calcification perpendicular to the longitudinal direction of the vessel) of the calcification $<2 / 3$ of vessel diameter, as previously described (Figure 1). ${ }^{17}$ Plaques with spotty calcification were further differentiated according to their length (extent of the calcification parallel to the longitudinal direction of the vessel on curved multiplanar reconstruction) into small spotty $(<1 \mathrm{~mm})$, intermediate spotty (1-3 mm), and large spotty calcifications $(\geq 3 \mathrm{~mm})$ as measured with caliper function (for illustration see Figure 2). ${ }^{18}$ Dense calcifications were defined as a plaque with high CT density, completely calcified and with calcifications present bilateral on cross-sectional axial slices.

\section{Invasive IVUS-VH}

Image acquisition. Conventional invasive coronary angiography was performed according to standard protocols. IVUS examinations were acquired with a $20 \mathrm{MHz}$, $2.9 \mathrm{~F}$ phased-array IVUS catheter, (Eagle Eye, Volcano Corporation, Rancho Cordova, CA, USA) and a dedicated IVUSconsole (Volcano Corporation, Rancho Cordova, CA, USA) after intracoronary administration of nitroglycerine. Under fluoroscopic guidance, the IVUS catheter was introduced distally in the coronary artery and a motorized automated pullback with a continuous speed of $0.5 \mathrm{~mm} / \mathrm{second}$ was used until the catheter reached the guiding catheter. Images were stored on CD-ROM or DVD for off-line analysis.

Image analysis. IVUS-VH analysis was performed by two experienced observers blinded to baseline patient characteristics and CTA findings with the use of dedicated software (QCU-CMS, version 4.59, Medis Medical Imaging Systems, Leiden, the Netherlands). First, contour detection (lumen and media interface) was performed manually with the use of cross-sectional views to provide compositional output. ${ }^{19}$ Secondly, on a frame-by-frame basis, the four different tissue components were differentiated and labeled with four different color-codes (fibrotic tissue was labeled in dark green, fibrofatty tissue in light green, necrotic core in red and dense calcium in white), as validated previously. ${ }^{10}$ The mean percentage of each plaque component was obtained in the full length of the lesion observed on the CTA examination. A lesion on intravascular ultrasound imaging was defined as at least three consecutive frames with a plaque burden of at least $40 \% .{ }^{20}$ In addition, the presence of the thin cap fibroatheroma (TCFA)
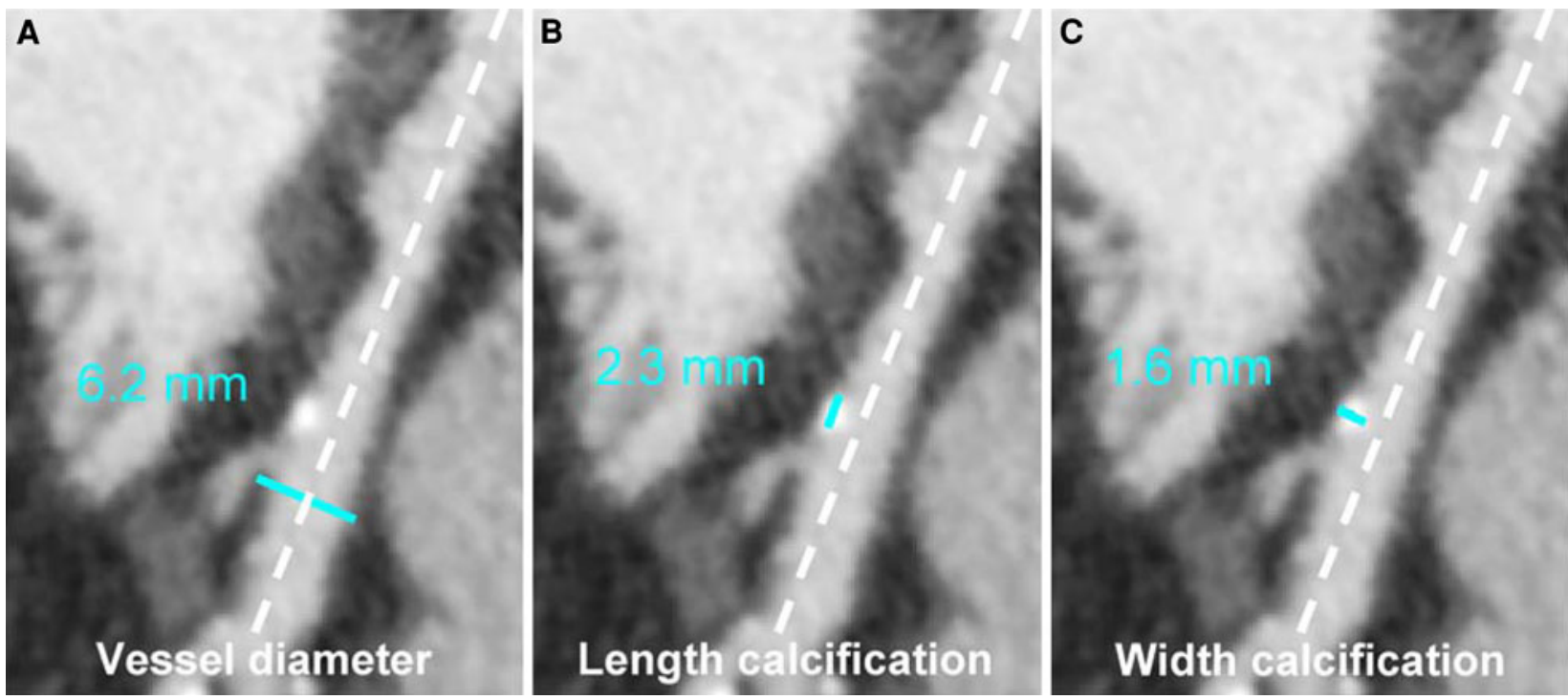

Figure 1. Example of measurement of spotty calcification. Plaques with spotty calcifications were defined as follows: length (extent in the longitudinal direction of the vessel) of the calcification $<3 / 2$ of vessel diameter and width (extent of the calcification perpendicular to the longitudinal direction of the vessel) of the calcification $<2 / 3$ of vessel diameter. A. Measurement of vessel diameter (blue line) perpendicular to long vessel axis (dashed white line). $\mathbf{B}$, Measurement of length of calcification (blue line) parallel to long vessel axis (dashed white line). $\mathbf{C}$, Measurement of width of calcification (blue line) perpendicular to long vessel axis (dashed line). 

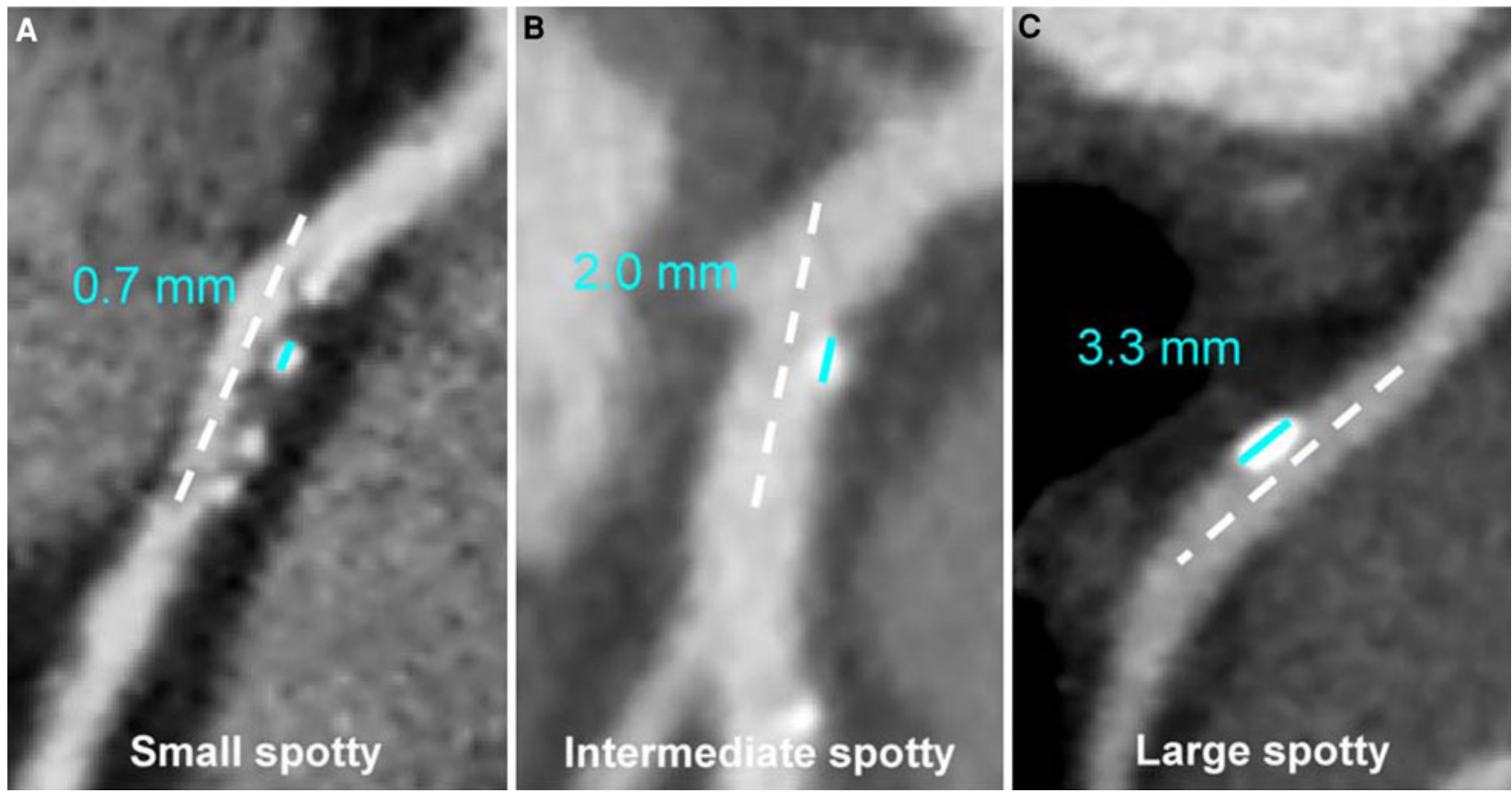

Figure 2. Illustration of the different types of spotty calcification. A, Small spotty calcification $(<1 \mathrm{~mm})$. B, Intermediate spotty calcification $(1-3 \mathrm{~mm})$. C, Large spotty calcification $(>3 \mathrm{~mm})$.

was evaluated on a per-lesion basis. TCFA was defined as a lesion with a plaque burden $\geq 40 \%$, presence of $>10 \%$ confluent necrotic core on three consecutive frames and necrotic core in contact with the lumen for along the lumen circumference. ${ }^{14,21,22}$ High-risk plaque characteristics on IVUS$\mathrm{VH}$ were defined by $\%$ of necrotic core and presence of TCFA.

To ensure that identical plaques were assessed by CTA and IVUS-VH, coronary ostia and side branches were used as landmarks and distances from the landmarks to the target lesions were measured (Figure 3). On CTA, distances were measured using multiplanar reconstructions. On IVUS-VH, longitudinal reconstructed ECG triggered datasets were used to measure the difference between corresponding plaque and landmarks. After alignment of plaques on CTA and IVUS-VH, we identified matching slices between the two modalities and could determine the proximal and distal end of a lesion. Examples of different calcification patterns on CTA with corresponding IVUS-VH images are demonstrated in Figures 4 and 5 .

Statistical analysis. Plaques available on both CTA and IVUS-VH were included in the lesion based analysis. Plaque composition (\% fibrotic, fibro-fatty, necrotic core, and dense calcium) and presence of TCFA was compared between lesions with different calcification patterns on CTA (non-calcified, small spotty calcifications, intermediate spotty calcifications, large spotty calcifications, and dense calcifications). If normally distributed, continuous values were expressed as means ( \pm standard deviation) and differences in plaque composition and type were assessed using an analysis of variance (ANOVA). If not normally distributed, values were expressed as medians (interquartile range (IQR)) and differences in plaque composition and type were assessed using the Kruskall-Wallis test. To account for possible clustering of coronary artery plaques within patients, the generalized estimating equation (GEE) method was applied to evaluate the differences in plaque characteristics between the groups of different calcification patterns on CTA (noncalcified, small spotty calcifications, intermediate spotty calcifications, large spotty calcifications, and dense calcium). This was performed with proc GENMOD with a binominal distribution for the outcome variable, the link function specified as a logistical (presence of TCFA) or a linear (\% fibrotic, fibrofatty, necrotic core, and dense calcium) distribution and patients as separate subjects. Due to lack of power, we did not perform post-hoc analysis. A $P$ value of $<.05$ was considered statistically significant. Statistical analysis was performed using SPSS 17.0 software (SPSS Inc., Chicago. Illinois).

\section{RESULTS}

Out of a total of 108 patients, 33 patients (31\%) were scanned with a 64-row CTA scanner and 75 patients $(69 \%)$ with a 320-row CTA scanner. Baseline patient characteristics are provided in Table 1. In summary, 80 patients $(74 \%)$ were male and mean age was $57 \pm 10$ years. IVUS-VH examination was successfully performed in 264 vessels $(81 \%)$ of the available 324 vessels without complications (right coronary artery (RCA), $\mathrm{n}=88$; left anterior descending coronary artery (LAD), $\mathrm{n}=98$; left circumflex coronary artery (LCx), $\mathrm{n}=78$ ). In the remaining vessels, IVUS imaging could 

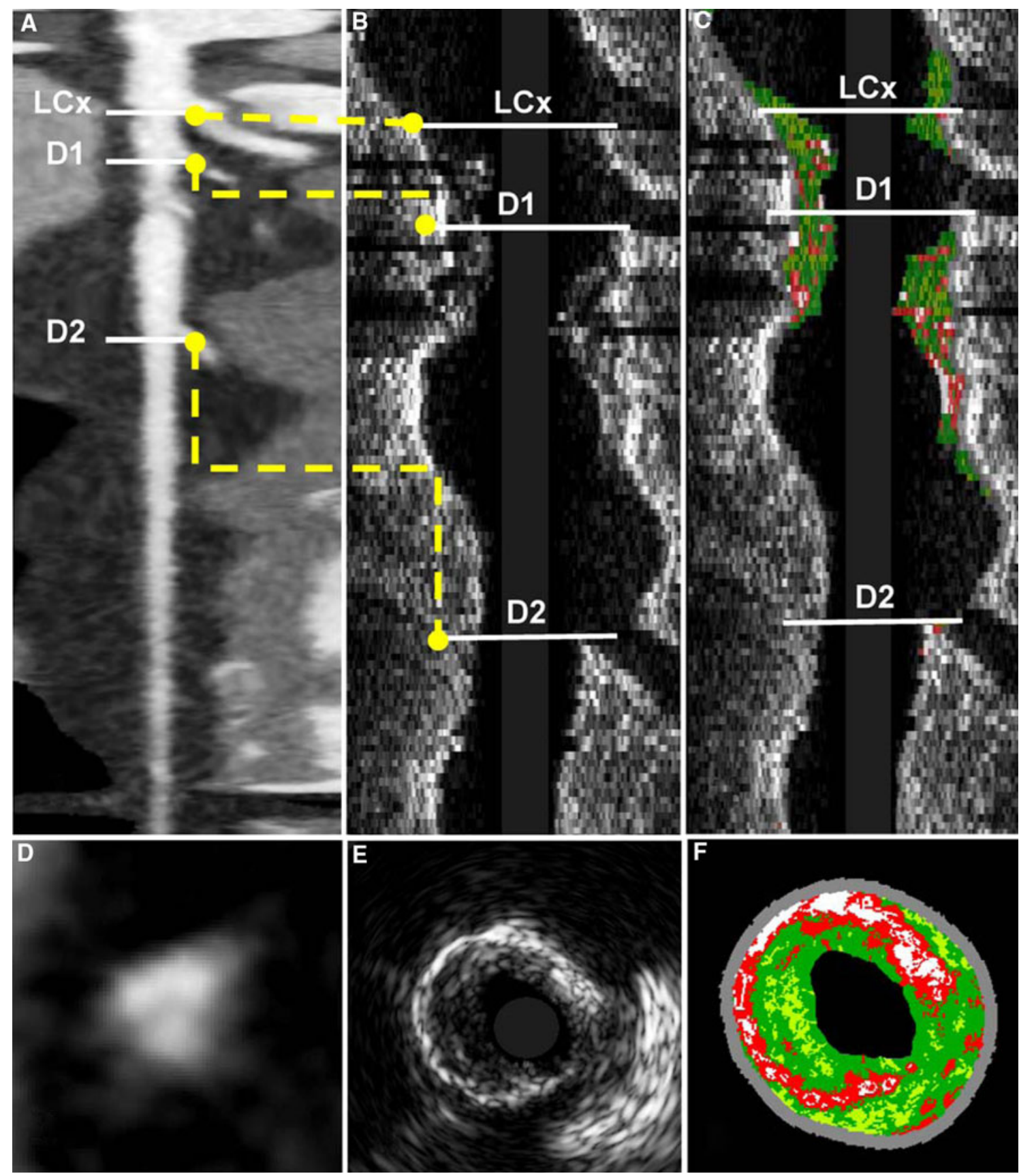

Figure 3. Example of anatomical matching of computed tomography angiography (CTA) with intravascular ultrasound with radiofrequency backscatter analysis (IVUS-VH) datasets. A, Straightened multiplanar reconstruction of left anterior descending coronary artery in which various landmarks have been identified (left circumflex artery (LCx), first and second diagonal branch (D1 and D2). B and C, Corresponding grayscale intravascular ultrasound and IVUS-VH datasets in longitudinal view in which identical landmarks have been matched to CTA dataset (yellow dashed line). Corresponding cross-sectional view of minimal lumen area on CTA (D) and IVUS-VH (E, F).

not be performed due to severe vessel tortuousity, severe stenosis, vessel occlusion or due to time-constraints in the cathlab.

\section{Baseline CTA and IVUS-VH Results}

In total, 799 plaques were demonstrated on CTA and in 300 plaques (38\%) IVUS-VH was also available. 

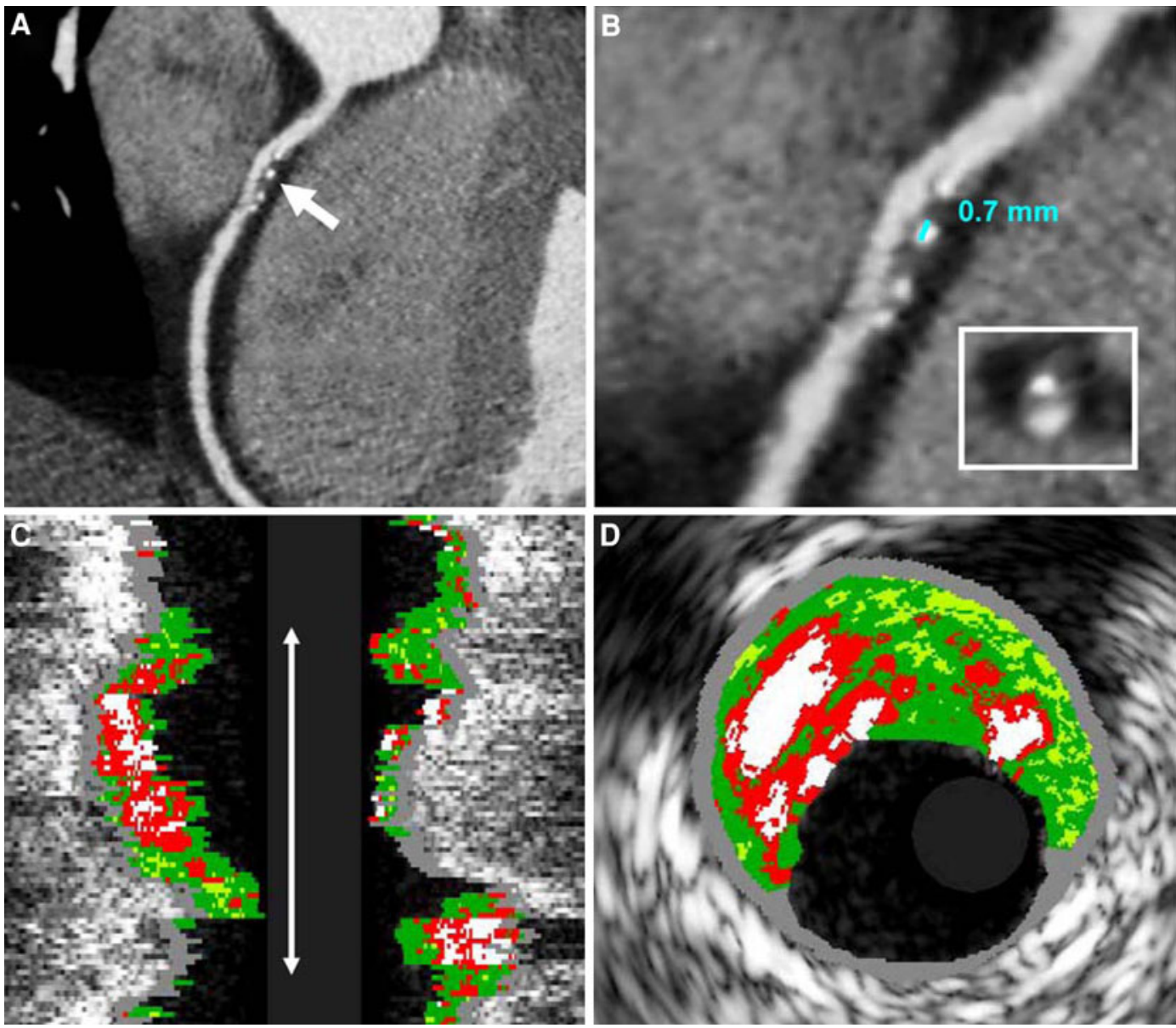

Figure 4. Example of small spotty calcifications as assessed on computed tomography angiography (CTA) with high-risk plaque characteristics on intravascular ultrasound with radiofrequency backscatter analysis (IVUS-VH). A, Multiplanar reconstruction of the right coronary artery (RCA) demonstrating a plaque with non-calcified elements and spotty calcifications (arrow). B, Enlargement of the same plaque demonstrating a spotty calcification with a length of $0.7 \mathrm{~mm}$. C, Longitudinal reconstructed ECG triggered IVUS-VH run with superimposed color coded map. The plaque (white arrow) corresponds to the same plaque on CTA demonstrating a large necrotic core and multiple small spotty calcifications. D, Cross-sectional axial image of the same plaque demonstrating calcifications (white) and a large necrotic core (red) indicating presence of a thin cap fibroatheroma (TCFA).

Of the 300 plaques identified on CTA, 78 plaques $(26 \%)$ were non-calcified, 39 plaques $(13 \%)$ had small spotty calcifications $(<1 \mathrm{~mm}), 96$ plaques $(32 \%)$ had intermediate spotty calcifications $(1-3 \mathrm{~mm}), 54$ plaques $(18 \%)$ had large spotty calcifications $(\geq 3 \mathrm{~mm})$, and 33 plaques $(11 \%)$ had dense calcifications. On IVUS-VH, the average plaque length analyzed was $26 \pm 18 \mathrm{~mm}$. The most prevalent plaque component was fibrotic tissue (58\%, IQR 50\%-63\%), followed by necrotic core $(15 \%$, IQR $10 \%-21 \%$ ), fibro-fatty tissue (13\%, IQR $8 \%-21 \%$ ), and dense calcium (6\%, IQR 3\%-10\%). Visual plaque assessment revealed that 52 plaques $(17 \%)$ were classified as TCFA.

\section{Comparison of Calcification Patterns on CTA and Plaque Composition on IVUS-VH}

The results comparing calcification patterns on CTA against relative plaque composition on IVUS-VH are reported in Table 2. Small spotty calcifications contained less fibro-fatty tissue (9\%, IQR $6 \%-15 \%$ ) as compared to non-calcified plaques (12\%, IQR $8 \%-22 \%)$, intermediate spotty calcifications (13\%, IQR 7\%21\%), large spotty calcifications (15\%, IQR 9\%-25\%), and dense calcifications (20\%, IQR $13 \%-25 \%, P<.05)$. In line with this observation, the more calcified plaques 

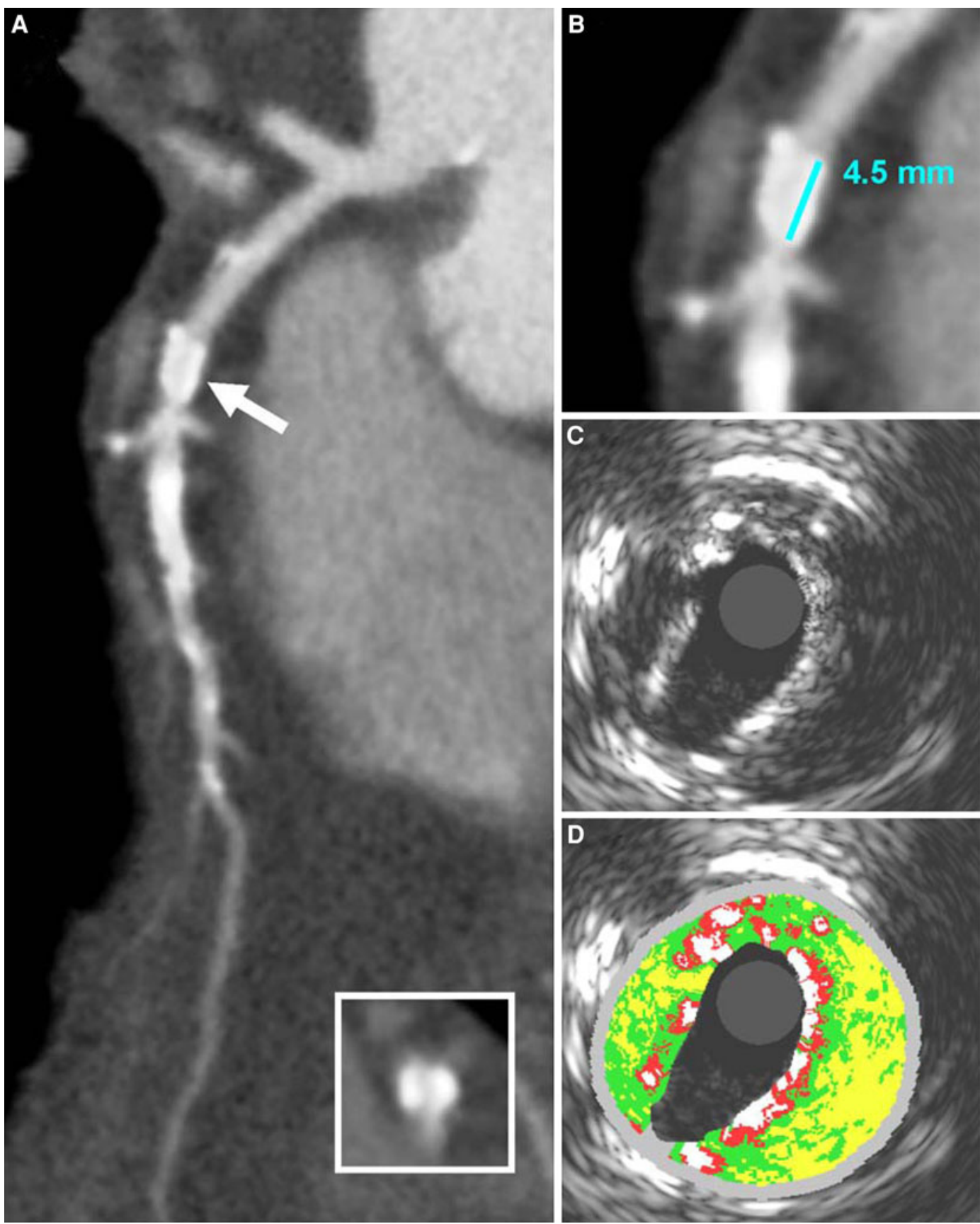

Figure 5. Example of a plaque with dense calcifications on computed tomography angiography (CTA) with corresponding grayscale and intravascular ultrasound with radiofrequency backscatter analysis (IVUS-VH) images. A, Curved multiplanar reconstruction of the right coronary artery (RCA) demonstrating a plaque with dense calcifications (arrow). Inlay showing cross-sectional axial image of the same plaque with calcifications present bilateral of the coronary artery. B, Enlargement of the same plaque reporting the length of the calcification $(4.5 \mathrm{~mm})$. C, Grayscale IVUS image demonstrating a heavily calcified plaque with corresponding IVUS-VH image (D) showing a plaque with heavy calcifications (white).

contained significantly more dense calcium on IVUS$\mathrm{VH}$ than the non-calcified plaques (9\%, IQR $6 \%-11 \%$ vs $3 \%$, IQR $1 \%-6 \%, P<.001)$. More importantly, necrotic core was significantly higher in plaques with small spotty calcifications (20\%, IQR $12 \%-24 \%$ ) as compared to non-calcified plaques (13\%, IQR 6\%-20\%, $P=.006$ ). 
Table 1. Baseline patient characteristics

\begin{tabular}{ll}
\hline Number of patients & 108 \\
Age (years) & $57 \pm 10$ \\
Male gender & $80(74 \%)$ \\
Stable angina/suspected acute coronary & $38 / 70$ \\
$\quad$ syndrome & \\
Hypertension & \\
Hypercholesterolemia ${ }^{\dagger}$ & $63(58 \%)$ \\
Diabetes & $55(51 \%)$ \\
Smoking & $29(27 \%)$ \\
Family history of CAD & $43(40 \%)$ \\
Obesity ( $\left.\geq 30 \mathrm{~kg}^{2}{ }^{2}\right)$ & $49(45 \%)$ \\
Medication at time of referral & $21(19 \%)$ \\
Aspirin & \\
Statins & $61(57 \%)$ \\
ACE inhibitor & $69(64 \%)$ \\
Beta-blockers & $53(49 \%)$ \\
Previous myocardial infarction & $58(54 \%)$ \\
Previous PCI & $22(20 \%)$ \\
\end{tabular}

Data are absolute values, percentages or means \pm standard deviation

$C A D$, Coronary artery disease; $A C E$, angiotensin-converting enzyme; $\mathrm{PCl}$, percutaneous coronary intervention

$\dagger$ Defined as systolic blood pressure $\geq 140 \mathrm{mmHg}$ or diastolic blood pressure $\geq 90 \mathrm{mmHg}$ or the use of antihypertensive medication

\$Serum total cholesterol $\geq 230 \mathrm{mg} / \mathrm{dL}$ or serum triglycerides $\geq 200 \mathrm{mg} / \mathrm{dL}$ or treatment with lipid lowering drugs

In addition, there was a trend for a higher percentage of necrotic core in plaques with small spotty calcifications than in plaques with intermediate spotty calcifications $(P=.053)$. Moreover, as demonstrated in Figure 6, plaques with small spotty calcifications were demonstrated to have a high percentage of TCFA $(31 \%)$ as compared to large spotty calcifications $(9 \%)$ and dense calcifications $(6 \%, P<.05)$. In addition, there was a trend for plaques with small spotty calcifications to have a higher percentage of TCFA than plaques with intermediate spotty calcifications $(17 \%, \quad P=.073)$. However, no significant difference was demonstrated between the percentage of TCFA in plaques with small spotty calcifications and non-calcified plaques $(P=.37)$.

\section{DISCUSSION}

This study systematically evaluated the relation between different calcification patterns on CTA and plaque characteristics on invasive IVUS-VH. It was demonstrated that plaques with spotty calcifications, in particular the smaller spotty calcifications $(<1 \mathrm{~mm})$ had plaque characteristics deemed high-risk on IVUS-VH, as defined by a higher $\%$ of necrotic core and presence of TCFA.

Interestingly, the present findings are in line with histopathological studies which have reported that lesions associated with acute coronary events are often not heavily calcified. ${ }^{20,21,23}$ Burke et $\mathrm{al}^{21}$ studied the degree of calcification in serial sections of coronary arteries in sudden coronary death cases and observed that plaque ruptures showed relatively little calcification and that most acute plaque ruptures resulting in sudden death occurred in areas of only mild speckled calcification. Furthermore, smaller calcium deposits on grayscale IVUS have been related to more unstable clinical presentation such as unstable angina and myocardial infarction. Beckman et $\mathrm{al}^{24}$ measured the arc of calcium in lesions of 78 patients and found that the average arc of calcium was greatest $\left(32^{\circ} \pm 7^{\circ}\right)$ in patients with stable angina, less $\left(15^{\circ} \pm 4^{\circ}\right)$ in patients with unstable angina, and least $\left(10^{\circ} \pm 5^{\circ}\right)$ in patients with acute myocardial infarction $(P<.014)$. In line with these findings, van der Hoeven et $\mathrm{al}^{18}$ studied 60 patients

Table 2. Plaque composition on intravascular ultrasound with radiofrequency backscatter analysis (IVUS-VH) is reported in relation to the different calcification patterns on computed tomography angiography (CTA)

\begin{tabular}{lcccccr}
\hline & $\begin{array}{c}\text { Non- } \\
\text { calcified }\end{array}$ & $\begin{array}{c}\text { Small } \\
\text { spotty } \\
(<\mathbf{1} \text { mm) }\end{array}$ & $\begin{array}{c}\text { Intermediate } \\
\text { spotty } \\
(\mathbf{1} \text {-3 } \mathbf{~ m m})\end{array}$ & $\begin{array}{c}\text { Large } \\
\text { spotty } \\
(\geq 3 \mathbf{~ m m})\end{array}$ & $\begin{array}{c}\text { Dense } \\
\text { calcifications }\end{array}$ & $\begin{array}{r}\text { Global } \\
\text { P value }\end{array}$ \\
\hline \% Fibrotic & $61(55-68)$ & $56(50-63)$ & $58(50-64)$ & $54(49-59)$ & $54(49-59)$ & $<.001$ \\
\% Fibro-fatty & $12(8-22)^{*}$ & $9(6-15)$ & $13(7-21)^{*}$ & $15(9-25)^{*}$ & $20(13-25)^{*}$ & $<.001$ \\
$\%$ Necrotic core & $13(6-20)^{*}$ & $20(12-24)$ & $14(9-21)^{*}$ & $17(13-21)$ & $14(12-22)$ & .003 \\
$\%$ Dense calcium & $3(1-6)^{*}$ & $7(4-13)$ & $6(3-10)$ & $9(6-13)$ & $9(6-11)$ & $<.001$ \\
\hline
\end{tabular}

Data in parentheses are percentages with $95 \%$ confidence intervals

* $P$ value $<.05$, individual groups compared to plaques with small spotty calcifications as reference group

${ }^{*} P$ value $=.053$, individual group compared to plaques with small spotty calcifications as reference group 


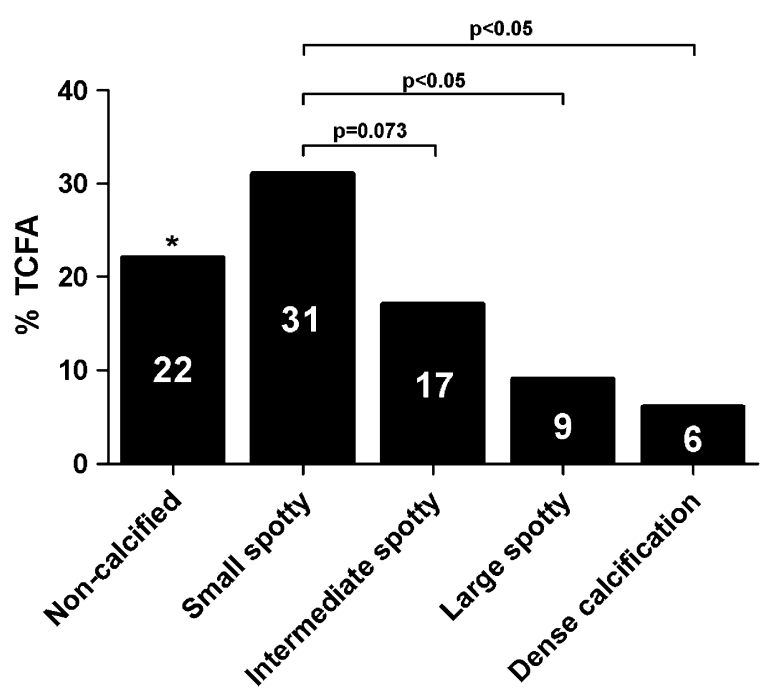

Figure 6. Bar graph represents the percentage of thin cap fibroatheroma (TCFA) in relation to the different calcification patterns on computed tomography angiography (CTA). As is shown, in particular plaques with small spotty calcifications on CTA contain the highest percentage of TCFA as compared to large spotty calcifications and dense calcium. *Small spotty calcifications did not have more TCFA than non-calcified plaques $(P=\mathrm{ns})$.

with acute myocardial infarction on grayscale IVUS and demonstrated that small calcified spots (arc $<45^{\circ}$, length $<1.5 \mathrm{~mm}$ ) were more common in the culprit lesions as compared to the adjacent distal and proximal segments. Accordingly, Ehara et $\mathrm{al}^{25}$ confirmed the findings in a larger patient population of 178 patients with grayscale IVUS. The authors found that not only the frequency of calcium deposits was significantly different between patients with stable, unstable angina, and myocardial infarction but also calcium deposits were significantly longer in patients with stable angina (mean length of $4.3 \pm 3.2 \mathrm{~mm}$ ) as compared to patients with unstable angina (mean length of $1.9 \pm 1.8$ ) and myocardial infarction (mean length of $2.2 \pm 1.6$ ). Thus, these findings support the concept that there may be a significant difference in the pattern of coronary calcifications, particularly with respect to size and length of the deposits, among patients with stable and unstable angina.

Non-invasively, calcium scoring with electron beam computed tomography (EBCT) showed similar findings. Although a higher calcium score has been related to larger plaque areas and worse prognosis, ${ }^{26}$ when comparing coronary calcium scores of patients with stable angina to patients with acute myocardial infarction, significant differences were observed. ${ }^{27}$ Extensive calcium was more often present in the coronary arteries of patients with chronic stable angina, whereas patients with acute myocardial infarction demonstrated a more diffuse pattern of calcifications. More interestingly, using contrastenhanced CTA, the presence of spotty calcifications were frequently present in lesions of patients with ACS but rarely observed in culprit lesions of patients with stable CAD. ${ }^{9}$ Similarly, Motoyama et al ${ }^{7,8}$ demonstrated both retrospectively and prospectively with CTA that noncalcified plaques with spotty calcifications and positive remodeling were associated with a higher likelihood of the development of ACS. However, unlike this study, the aforementioned findings were not related to invasive observations. Interestingly, a previous study by our institution evaluated the association between plaque composition on CTA and the presence of high-risk features on IVUS-VH in a smaller population of 50 patients. ${ }^{28}$ It was demonstrated that the more high-risk plaques (TCFA) were most often present in mixed plaques $(32 \%)$ on CTA. Although similar to the current findings, the $\%$ of TCFA in mixed plaques was higher in the previous evaluation than in the current study $(31 \%)$. This could probably be explained by difference in the size and baseline characteristics of the patient population. In addition, the amount of dense calcium in the current study is somewhat lower than reported in other studies. However, this could be explained by the inclusion of a substantial number $(65 \%)$ of patients presenting with acute coronary syndrome, known to have substantially less calcified arteries.

Interestingly, earlier investigations have used various definitions of spotty calcifications on CTA. For instance, some investigations described spotty calcification as any calcified material embedded within a non-calcified plaque, whereas other use a threshold of calcified material less than $3 \mathrm{~mm}$ in size. ${ }^{7,9,29}$ However, a comprehensive approach for the evaluation of the extent of the spotty calcifications on CTA is preferred, thereby improving reproducibility and use in risk stratification. The strength of the current study is that a systematic approach for the evaluation of spotty calcifications was applied, demonstrating that indeed the smaller spotty calcifications were related to the plaque characteristics deemed more high-risk such as necrotic core and presence of TCFA.

Importantly, the findings are clinically relevant considering that CTA is increasingly used for the assessment of CAD and the use of this technique is extended from assessment of stenosis to the evaluation of the vessel wall. Nevertheless, even with the latest generation CTA scanners, exact identification of the lipid core and thin fibrous cap is not feasible at the moment. Therefore, assessment of spotty calcification on CTA may be beneficial for individualized risk stratification in combination with other high-risk features such as cardiovascular risk factors and biomarkers. Perhaps, CTA has a potential application in the context 
of identifying the "vulnerable" patient at risk for ACS. However, the clinical and prognostic impact of these findings has to be evaluated in future prospective studies.

\section{LIMITATIONS}

First, due to the limitations of intravascular ultrasound, not all plaques on CTA were also evaluated by IVUS-VH. In addition, detection of the thin fibrous cap $(<65 \mu \mathrm{m})$ is not yet feasible as IVUS-VH has a limited radial resolution of only $100 \mu \mathrm{m}$. Furthermore, it has been suggested that dense calcium on IVUS-VH is related to artifacts in the form of a halo of necrotic core surrounding dense calcium. However, no standardized method for correcting for this issue is currently available. Additionally, no other high risk plaque characteristics were assessed on CTA. Potentially, incorporating other CTA variables such as degree of stenosis and overall plaque burden, may allow for better risk stratification. In addition, this study performed a retrospective analysis of data possibly introducing a bias with regards to lesion selection. Future evaluations examining on the relationship between calcification patterns on CTA and IVUS-VH characteristics should be performed in a more prospective manner. Moreover, reproducibility information on the measurement of calcification on CTA was not provided. Lastly, CTA is inherently associated with radiation exposure. However, recently, dose-saving algorithms and prospective ECG triggering were introduced to substantially reduce radiation dose. ${ }^{30}$

\section{CONCLUSION}

The current study demonstrated that plaques with small spotty calcifications on CTA were related to plaque characteristics deemed more high-risk on invasive IVUS-VH. Therefore, CTA may be valuable in the assessment of the vulnerable plaque.

\section{Acknowledgments}

Dr Schalij has research grants from Boston Scientific (Natick, USA), Medtronic (Minneapolis, United States) and Biotronik (Berlin, Germany). Dr Jukema receives grants from Biotronik (Berlin, Germany), Boston Scientific (Natick, USA), Astra Zeneca (London, United Kingdom), Pfizer (New York, United States), MSD (New Jersey, United States). Dr Bax has research grants from Medtronic (Minneapolis, United States), Boston Scientific (Natick, United States), BMS medical imaging (New York, United States), St. Jude Medical (St. Paul, United States), GE Healthcare (Chalfont St Giles,
United Kingdom), Biotronik (Berlin, Germany), and Edwards Lifesciences (Irvine, United States).

\section{Open Access}

This article is distributed under the terms of the Creative Commons Attribution Noncommercial License which permits any noncommercial use, distribution, and reproduction in any medium, provided the original author(s) and source are credited.

\section{References}

1. Achenbach S, Giesler T, Ropers D, Ulzheimer S, Derlien H, Schulte C, et al. Detection of coronary artery stenoses by contrastenhanced, retrospectively electrocardiographically-gated, multislice spiral computed tomography. Circulation 2001;103:2535-8.

2. Budoff MJ, Dowe D, Jollis JG, Gitter M, Sutherland J, Halamert E, et al. Diagnostic performance of 64-multidetector row coronary computed tomographic angiography for evaluation of coronary artery stenosis in individuals without known coronary artery disease: Results from the prospective multicenter ACCURACY (Assessment by Coronary Computed Tomographic Angiography of Individuals Undergoing Invasive Coronary Angiography) trial. J Am Coll Cardiol 2008;52:1724-32.

3. Leber AW, Knez A, Becker A, Becker C, von ZF, Nikolaou K, et al. Accuracy of multidetector spiral computed tomography in identifying and differentiating the composition of coronary atherosclerotic plaques: A comparative study with intracoronary ultrasound. J Am Coll Cardiol 2004;43:1241-7.

4. Miller JM, Rochitte CE, Dewey M, rbab-Zadeh A, Niinuma H, Gottlieb I, et al. Diagnostic performance of coronary angiography by 64-row CT. N Engl J Med 2008;359:2324-36.

5. Van Velzen JE, Schuijf JD, De Graaf FR, Boersma E, Pundziute G, Spano F, et al. Diagnostic performance of non-invasive multidetector computed tomography coronary angiography to detect coronary artery disease using different endpoints: Detection of significant stenosis vs. detection of atherosclerosis. Eur Heart $\mathbf{J}$ 2011;32:637-45.

6. Hoffmann U, Moselewski F, Nieman K, Jang I, Ferencik M, Rahman A. Noninvasive assessment of plaque morphology and composition in culprit and stable lesions in acute coronary syndrome and stable lesions in stable angina by multidetector computed tomography. J Am Coll Cardiol 2006;47:1655-62.

7. Motoyama S, Kondo T, Sarai M, Sugiura A, Harigaya H, Sato T, et al. Multislice computed tomographic characteristics of coronary lesions in acute coronary syndromes. J Am Coll Cardiol 2007;50: 319-26.

8. Motoyama S, Sarai M, Harigaya H, Anno H, Inoue K, Hara T, et al. Computed tomographic angiography characteristics of atherosclerotic plaques subsequently resulting in acute coronary syndrome. J Am Coll Cardiol 2009;54:49-57.

9. Pflederer T, Marwan M, Schepis T, Ropers D, Seltmann M, Muschiol G, et al. Characterization of culprit lesions in acute coronary syndromes using coronary dual-source CT angiography. Atherosclerosis 2010;211:437-44.

10. Nasu K, Tsuchikane E, Katoh O, Vince DG, Virmani R, Surmely $\mathrm{JF}$, et al. Accuracy of in vivo coronary plaque morphology assessment: A validation study of in vivo virtual histology compared with in vitro histopathology. J Am Coll Cardiol 2006;47: 2405-12. 
11. Pundziute G, Schuijf JD, Jukema JW, van Werkhoven JM, Nucifora G, Decramer I, et al. Type 2 diabetes is associated with more advanced coronary atherosclerosis on multislice computed tomography and virtual histology intravascular ultrasound. J Nucl Cardiol 2009;16:376-83.

12. Nair A, Kuban DB, Tuzcu EM, Schoenhagen P, Nissen SE, Vince DG. Coronary plaque classification with intravascular ultrasound radiofrequency data analysis. Circulation 2002;106:2200-6.

13. Voros S, Rinehart S, Qian Z, Vazquez G, Anderson H, Murrieta L, et al. Prospective validation of standardized, 3-dimensional, quantitative coronary computed tomographic plaque measurements using radiofrequency backscatter intravascular ultrasound as reference standard in intermediate coronary arterial lesions: Results from the ATLANTA (assessment of tissue characteristics, lesion morphology, and hemodynamics by angiography with fractional flow reserve, intravascular ultrasound and virtual histology, and noninvasive computed tomography in atherosclerotic plaques) I study. JACC Cardiovasc Interv 2011;4:198-208.

14. Stone GW, Maehara A, Lansky AJ, De BB, Cristea E, Mintz GS, et al. A prospective natural-history study of coronary atherosclerosis. N Engl J Med 2011;364:226-35.

15. Van Velzen JE, Schuijf JD, De Graaf FR, Nucifora G, Pundziute G, Jukema JW, et al. Plaque type and composition as evaluated non-invasively by MSCT angiography and invasively by $\mathrm{VH}$ IVUS in relation to the degree of stenosis. Heart 2009;95:1990-6.

16. Austen WG, Edwards JE, Frye RL, Gensini GG, Gott VL, Griffith LS, et al. A reporting system on patients evaluated for coronary artery disease. Report of the Ad Hoc Committee for Grading of Coronary Artery Disease, Council on Cardiovascular Surgery, American Heart Association. Circulation 1975;51:5-40.

17. Kitagawa T, Yamamoto H, Horiguchi J, Ohhashi N, Tadehara F, Shokawa T, et al. Characterization of noncalcified coronary plaques and identification of culprit lesions in patients with acute coronary syndrome by 64 -slice computed tomography. JACC Cardiovasc Imaging 2009;2:153-60.

18. van der Hoeven BL, Liem SS, Oemrawsingh PV, Dijkstra J, Jukema JW, Putter H, et al. Role of calcified spots detected by intravascular ultrasound in patients with ST-segment elevation acute myocardial infarction. Am J Cardiol 2006;98:309-13.

19. Mintz GS, Nissen SE, Anderson WD, Bailey SR, Erbel R, Fitzgerald PJ, et al. American College of Cardiology Clinical Expert Consensus Document on Standards for Acquisition, Measurement and Reporting of Intravascular Ultrasound Studies (IVUS). A report of the American College of Cardiology Task Force on
Clinical Expert Consensus Documents. J Am Coll Cardiol 2001;37:1478-92.

20. Virmani R, Kolodgie FD, Burke AP, Farb A, Schwartz SM. Lessons from sudden coronary death: A comprehensive morphological classification scheme for atherosclerotic lesions. Arterioscler Thromb Vasc Biol 2000;20:1262-75.

21. Burke AP, Weber DK, Kolodgie FD, Farb A, Taylor AJ, Virmani R. Pathophysiology of calcium deposition in coronary arteries. Herz 2001;26:239-44.

22. Carlier SG, Mintz GS, Stone GW. Imaging of atherosclerotic plaque using radiofrequency ultrasound signal processing. J Nucl Cardiol 2006;13:831-40.

23. Virmani R, Burke AP, Kolodgie FD, Farb A. Pathology of the thin-cap fibroatheroma: A type of vulnerable plaque. J Intervent Cardiol 2003; 16:267-72.

24. Beckman JA, Ganz J, Creager MA, Ganz P, Kinlay S. Relationship of clinical presentation and calcification of culprit coronary artery stenoses. Arterioscler Thromb Vasc Biol 2001;21:1618-22.

25. Ehara S, Kobayashi Y, Yoshiyama M, Shimada K, Shimada Y, Fukuda D, et al. Spotty calcification typifies the culprit plaque in patients with acute myocardial infarction: An intravascular ultrasound study. Circulation 2004;110:3424-9.

26. Detrano R, Guerci AD, Carr JJ, Bild DE, Burke G, Folsom AR, et al. Coronary calcium as a predictor of coronary events in four racial or ethnic groups. N Engl J Med 2008;358:1336-45.

27. Shemesh J, Stroh CI, Tenenbaum A, Hod H, Boyko V, Fisman EZ, et al. Comparison of coronary calcium in stable angina pectoris and in first acute myocardial infarction utilizing double helical computerized tomography. Am J Cardiol 1998;81:271-5.

28. Pundziute G, Schuijf JD, Jukema JW, Decramer I, Sarno G, Vanhoenacker PK, et al. Head-to-head comparison of coronary plaque evaluation between multislice computed tomography and intravascular ultrasound radiofrequency data analysis. JACC Cardiovasc Interv 2008; 1:176-82.

29. Utsunomiya H, Yamamoto H, Kunita E, Kitagawa T, Ohashi N, Oka $\mathrm{T}$, et al. Combined presence of aortic valve calcification and mitral annular calcification as a marker of the extent and vulnerable characteristics of coronary artery plaque assessed by 64-multidetector computed tomography. Atherosclerosis 2010;213:166-72.

30. Herzog BA, Wyss CA, Husmann L, Gaemperli O, Valenta I, Treyer V, et al. First head-to-head comparison of effective radiation dose from low-dose 64-slice CT with prospective ECGtriggering versus invasive coronary angiography. Heart 2009;95: 1656-61. 